\title{
Eigenfettinjektion in das Sattelgelenk zur Behandlung der Rhizarthrose - eine viel versprechende Therapieoption
}

\author{
Autologous Fat Injection for Treatment of Carpometacarpal Joint \\ Osteoarthritis of the Thumb - A Promising Alternative
}

Autoren

Institut

\author{
C. Herold, O. Fleischer, S. Allert
}

Klinik für Plastische und Ästhetische Chirurgie, Handchirurgie, Sana Klinikum Hameln Pyrmont, Hameln

\author{
Schlüsselwörter \\ - Arthrose \\ Resektions-Arthroplastik \\ - Daumensattelgelenk \\ - Daumen \\ - Fettgewebe
}

\section{Key words}

osteoarthritis

- resection arthroplasty

- thumb carpometacarpal joint

- thumb

- adipose tissue eingereicht 6.1.2014 akzeptiert 27.2.2014

\section{Bibliografie}

DOI http://dx.doi.org/ 10.1055/s-0034-1371819 Handchir Mikrochir Plast Chir 2014; 46: 108-112 (c) Georg Thieme Verlag KG Stuttgart - New York ISSN 0722-1819

\footnotetext{
Korrespondenzadresse PD Dr. Christian Herold Klinik für Plastische und Ästhetische Chirurgie Handchirurgie Sana Klinikum Hameln Pyrmont St. Maur Platz 11 31785 Hameln christianherold@gmx.de
}

\section{Zusammenfassung \\ $\nabla$}

Klassische chirurgische Operationstechniken zur Behandlung der Rhizarthrose führen zu einer unwiderruflichen Veränderung der Anatomie der Handwurzel. Auch die Arbeitsunfähigkeit und das Wiedererlangen der Handfunktion nach diesen Eingriffen dauert in der Regel 12-16 Wochen. Daher erscheint eine nicht ablative und gering invasive chirurgische Therapieoption der Sattelgelenksarthrose eine wünschenswerte Alternative. In einer Pilotstudie injizieren wir Fettgewebe in das arthrotisch veränderte Daumensattelgelenk. Die ersten Ergebnisse sind viel versprechend. Der durchschnittliche präoperative Schmerz nach VAS betrug 7,4 bei Belastung und 3,8 in Ruhe. Der durchschnittliche postoperative Schmerz nach einem Monat betrug 2,2 bei Belastung und 0 in Ruhe, sowie 2,4 und 0,8 nach 3 Monaten. Die Schmerzminderung einen Monat nach Injektion bei Belastung war statistisch signifikant $(p=0,042)$. Die Kraft des Grobgriffes betrug 78\% und des Pinch Griffes 74\% im Vergleich zu der gesunden Seite präoperativ. Die Kraft des Grobgriffes betrug $89 \%$ und die des Pinch Griffes 80\% im Vergleich zu der gesunden Seite einen Monat postoperativ und $93 \%$ bzw. $89 \% 3$ Monate postoperativ. Entsprechend reduzierte sich der DASH Score von präoperativ durchschnittlich 58 auf 36 nach einem bzw. 33 nach 3 Monaten. Die Verbesserungen der Handfunktion waren statistisch signifikant $(p=0,042$ und $p=0,043)$. Es traten keine Komplikationen auf und alle Patienten waren zufrieden. Diese vorläufigen Ergebnisse sind viel versprechend, da die Eigenfettinjektion auch im Falle eines möglichen Therapieversagens, anders als die meisten bekannten Operationstechniken, den Wechsel auf eine andere klassische Operationsalternative nicht ausschließt.

\section{Abstract \\ $\nabla$}

Classical surgical options for osteoarthritis of the thumb carpometacarpal joint irreversibly destroy the normal anatomy of the wrist. Although overall satisfaction rates with these procedures are high, time to achieve normal hand function and ability to work may take 12-16 weeks. Therefore a non-ablative less invasive surgical option would be interesting. We injected adipose tissue into the thumb carpometacarpal joint in a pilot study. Average preoperative pain according to a VAS was 7.4 in action and 3.8 during rest. It was reduced considerably to 2.2 in action and 0 during rest after 1 month and to 2.4 and 0.8 , respectively, 3 months after surgery. The reduction of pain in action was statistically significant 1 month after injection $(p=0.042)$. Average grip strength was $78 \%$ and pinch grip strength was $74 \%$ in comparison to the healthy side preoperatively, $89 \%$ and $80 \%$ one month postoperatively and $93 \%$ and $89 \%$, respectively, 3 months postoperatively. An average DASH score of 58 preoperative was reduced to 36 after 1 month and 33 after 3 months. The amelioration of hand function was statistically significant $(p=0.042$ and $p=0.043$ ). There were no side effects and all patients were satisfied. These preliminary results are promising. Adipose tissue injection seems to be an alternative to consider, especially as it does not exclude classical surgical options in cases of failure. 


\section{Einleitung

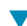

Die Daumensattelgelenksarthrose ist eine häufige Erkrankung, welche bis zu 25\% postmenopausaler Frauen betrifft [1]. Verschiedene konservative und operative Therapieoptionen stehen zur Verfügung. Falls eine konservative Behandlung frustran gewesen ist, können den Patienten chirurgische Alternativen wie eine metacarpale Extensionsosteotomie, eine Trapezektomie mit oder ohne Bandplastik, eine Trapeziometacarpale Arthrodese, eine Prothesenimplantation und verschiedene Formen der Interpositionsarthroplastiken [2], inklusive dermaler Matrix [3] angeboten werden.

Die operative Versorgung kann bei bis zu 95\% der Patienten ein zufriedenstellendes Ergebnis erzielen [2], dennoch zerstören die meistens Operationsmethoden die Anatomie der Handwurzel unwiderruflich.

Eine Verkürzung des Daumenstrahles, ein Kollaps des Karpus nach Trapezektomie oder eine Prothesendislokation sind einige Komplikationen, welche nach den klassischen Operationsmöglichkeiten der Rhizarthrose auftreten können [2]. Abhängig von der verwendeten Operationstechnik sind mehrwöchige Ruhigstellungen und ebenso lange physiotherapeutische Nachbehandlungen in der Regel notwendig. Die Arbeitsunfähigkeit nach Trapezium Resektionsarthroplastiken mit distal gestielter FCR Sehne in den Stadien $2-4^{\circ}$ nach Eaton und Littler wird bei Berufstätigen mit durchschnittlich 16 Wochen angegeben, wobei auf je 8 Wochen eine 100\% und 50\% Arbeitsunfähigkeit entfällt. Selbst Hausfrauen können erst nach 12 Wochen wieder alle Tätigkeiten im Haushalt ausüben [4].

Daher besteht die Notwendigkeit für dieses häufige Krankheitsbild eine gering invasive Alternative anbieten zu können, welche die Hauptprobleme eine Rhizarthrose: den Schmerz, die Kraftreduktion und die Einschränkung der Beweglichkeit des Daumens suffizient behebt, ohne lange Ausfallszeiten zu verursachen.

Die Injektion von Hyaluronsäure als temporäre Option zur Symptomreduktion der Sattelgelenksarthrose ist bereits beschrieben [5]. Körpereigenes Fett sollte dieselbe Pufferfunktion im verschlissenen Gelenk bieten können. Der hohe Gehalt an Vorläuferzellen im Fettgewebe ist wohlbekannt und auch deren Möglichkeit, sich in verschiedenste Gewebe, inklusive Knorpel, zu differenzieren $[6,7]$. In mehreren Studien konnte nicht nur ein antiinflammatorischer und chondroprotektiver Effekt von adipose derived stem cells nachweisen werden [8], es wurde auch das Potenzial von Fettstammzellen beschrieben, Knorpel regenerieren zu können [9]. Chondrozyten wiederum können eine Differenzierung von adipose derived stem cells anregen [9], sodass das Einbringen von Fettstammzellen in ein verschlissenes Gelenk prinzipiell sinnvoll erscheint.

Als Hypothese für diese Studie wird angenommen, dass die Isolierung von Fettstammzellen aus Lipoaspirat allerdings nicht notwendig ist, da auch normale Fetttransplantate Fettstammzellen enthalten und der Gleiteffekt des Fetttransplantates an sich bei der Therapie einer Arthrose von großem Interesse ist.

\section{Patienten, Material und Methoden}

\section{$\nabla$}

Nach Beantragung und Genehmigung durch die zuständige Ethikkommission begannen wir eine Studie zur Untersuchung der Effekte intraartikulärer Eigenfettinjektion in das Sattelgelenk bei Patienten mit klinisch und radiologisch relevanter Rhizarthrose. Es wurden Patienten eingeschlossen, bei denen eine konservative Therapie der Sattelgelenksarthrose frustran war, und welche in unserer Klinik bisher einer Trapezektomie zugeführt worden wären.

Wir berichten hier über die Ergebnisse der ersten 5 behandelten Patienten, allesamt Frauen mit einem mittleren Alter von 64 Jahren (48-83 Jahre). Es lagen 3-mal Sattelgelenksarthrosen Stadium $3^{\circ}$ und 2 -mal Stadium $2^{\circ}$ nach Eaton vor. In allen Fällen war die linke Seite betroffen.

Die Operationen wurden in Tumeszensanästhesie mit Kleinscher Lösung zur Absaugung am Abdomen und im Leitungsblock des Ramus superficialis operiert. Es erfolgte die Überwachung eine Nacht postoperativ stationär. Es wurde in klassischer Coleman Technik eine geringe Menge Fettgewebe vom Abdomen manuell in $10 \mathrm{ml}$ Luer Lock Spritzen aspiriert und für $3 \mathrm{~min}$ bei 3000 Umdrehungen/min in der Zentrifuge separiert. Es wurde die Coleman Nr. 1 Absaugkanüle verwendet. Nach Entfernen der Öl-, und Wasserphase erfolgte über eine Stichinzision über dem Daumensattelgelenk die intraartikuläre Injektion von $1,5 \mathrm{ml} \mathrm{Li}$ poaspirat unter Durchleuchtungskontrolle ( $\bullet$ Abb. 1, 2). Die Menge von $1,5 \mathrm{ml}$ ist das Volumen Fettgewebe, welches sich ohne Druck in das Sattelgelenk injizieren lässt. Wird versucht eine größere Menge zu injizieren, wird ein deutlich spürbarer Widerstand am Spritzenstempel bemerkbar. Der Daumen wurde hierbei unter axialem Zug gehalten, um den Gelenkspalt zu erweitern. Es wurde eine 14 G Einmalnadel zur Injektion verwendet. Der Hautverschluss erfolgte über eine Einzelknopfnaht und einen Steristrip Verband. Es wurde abschließend eine Unterarm-Daumenschiene angelegt, um den Daumenstrahl für 7 Tage ruhig zu stellen. Somit soll ein Dislozieren des injizierten Fettgewebes aus dem Daumensattelgelenk minimiert werden.

Die Patienten wurden nach 7 und 14 Tagen, sowie nach einem und 3 Monaten postoperativ im Rahmen der Studie nachuntersucht. Am siebten postoperativen Tag erfolgte das Ablegen der Schiene und die Wiederaufnahme der Alltagstätigkeiten ohne weitere Schonung. Eine physiotherapeutische Behandlung war bei keinem Patienten aufgrund der kurzen Ruhigstellung notwendig. Präoperativ, nach einem und nach 3 Monaten erfolgte die Evaluation über DASH Bögen.

Die Einstufung der Schmerzen erfolgte über eine VAS Skala (0-10) bei Belastung und in Ruhe. Die Grobkraft und der Pinch Griff wurden ebenfalls zu diesen Zeitpunkten bestimmt. Zusätzlich erfolgte eine Bestimmung der Daumenbeweglichkeit nach der Kapandji Skala. Die präoperativen Röntgenbilder wurden im

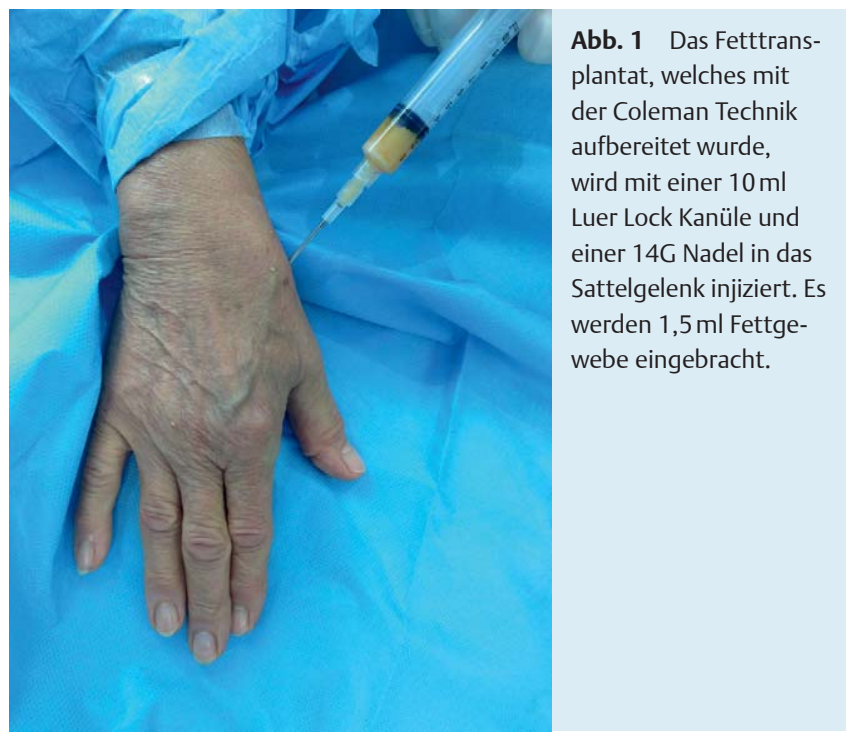


Rahmen der Studie um Röntgenbilder des Sattelgelenkes 3 Monate postoperativ ergänzt.

Eine statistische Auswertung erfolgte über einen Friedman Test und einen nachgeschalteten paarweise post hoc Test, den Wilcoxon Test. Als statistisch signifikant wurde ein p-Wert von $<0,05$ angesehen.

\section{Ergebnisse}

Der durchschnittliche präoperative Schmerz nach VAS betrug 7,4 bei Belastung und 3,8 in Ruhe. Der durchschnittliche postoperative Schmerz nach einem Monat betrug 2,2 bei Belastung

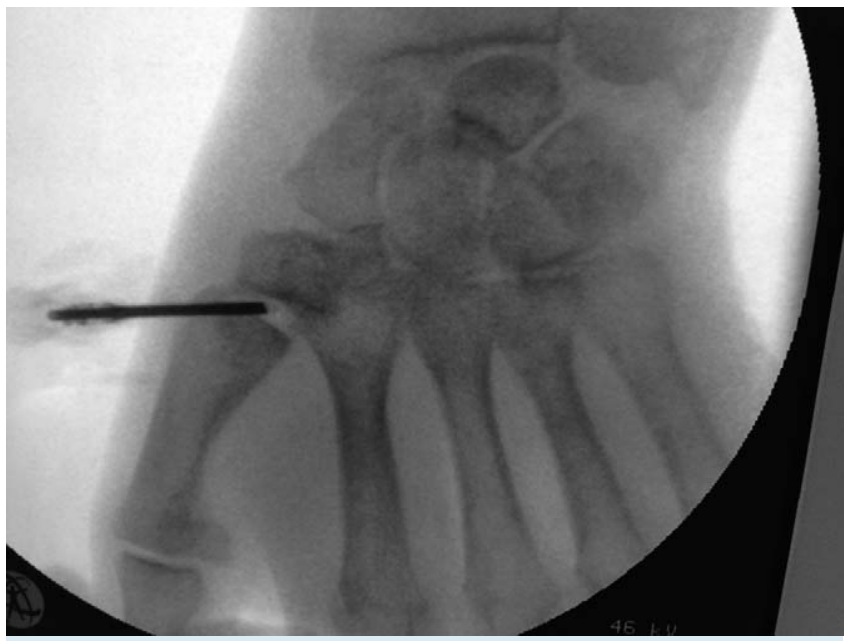

Abb. 2 Die Nadel wird unter Durchleuchtung in das Sattelgelenk eingebracht. Während der Punktion und der Injektion wird eine axiale Traktion auf den Daumen gebracht, um eine Traumatisierung des Gelenkes zu minimieren. und 0 in Ruhe, sowie 2,4 und 0,8 nach 3 Monaten. Es trat somit eine deutliche Schmerzreduktion ein, welche einen Monat postoperativ auch statistisch signifikant war $(p=0,042)$. Der Kapandji Score betrug sowohl präoperativ als auch zu beiden Zeitpunkten postoperativ durchschnittlich 10. $₫$ Tab. 1 fasst die demografischen Daten und Ergebnisse zusammen.

Die Kraft des Grobgriffes betrug 78\% und des Pinch Griffes 74\% im Vergleich zu der gesunden Seite präoperativ. Die Kraft konnte durch die Eigenfettinjektion verbessert werden. Die Kraft des Grobgriffes betrug 89\% und die des Pinch Griffes 80\% im Vergleich zu der gesunden Seite einen Monat postoperativ und 93\% bzw. 89\% 3 Monate postoperativ. Weder für den Pinch Griff noch für die Grobkraft waren die Verbesserungen statistisch signifikant, wenn auch für die Grobkraft grenzwertig $(p=0,059)$. Es traten keine Komplikationen auf und die Patienten würden den Eingriff wieder durchführen lassen.

Die Evaluation über die DASH Bögen bestätigte diese Ergebnisse. Präoperativ lag der DASH Wert bei durchschnittlich 58, einen Monat postoperativ bei 36 und 3 Monate postoperativ bei 33 . Somit zeigte sich sowohl einen $(p=0,042)$, als auch 3 Monate $(p=0,043)$ postoperativ eine signifikante Verbesserung der Einschätzung der Handfunktion durch die Patienten. Die Röntgenaufnahmen 3 Monate postoperativ zeigten keine intraartikulären Verkalkungen, eine wesentliche Veränderung der konventionellen radiologischen Befunde im Vergleich zu präoperativ war nicht festzustellen, das Beheben einer Subluxationsstellung war jedoch auch nicht zu erwarten.

\section{Diskussion}

Ziel dieser Studie war es, eine innovative nicht ablative Therapieoption der Sattelgelenksarthrose zu untersuchen, da klassi-

Tab. 1 Darstellung der demografischen Daten und Ergebnisse nach Fetttransplantation.

\begin{tabular}{|c|c|c|c|c|c|c|c|}
\hline Patient & 1 & 2 & 3 & 4 & 5 & & \\
\hline Geschlecht & $f$ & $f$ & $f$ & $f$ & $f$ & & \\
\hline Alter & 80 & 49 & 48 & 83 & 59 & & \\
\hline Stadium & $3^{\circ}$ & $2^{\circ}$ & $3^{\circ}$ & $3^{\circ}$ & $2^{\circ}$ & & \\
\hline Seite & links & links & links & links & links & & \\
\hline $\begin{array}{l}\text { bisher fustrane } \\
\text { konservative } \\
\text { Therapie }\end{array}$ & $\begin{array}{l}\text { Antiphl. Thera- } \\
\text { pie Sattelge- } \\
\text { lenks-Schiene }\end{array}$ & $\begin{array}{l}\text { Antiphl. Thera- } \\
\text { pie Sattelge- } \\
\text { lenks-Schiene }\end{array}$ & $\begin{array}{l}\text { Antiphl. Thera- } \\
\text { pie Sattelge- } \\
\text { lenks-Schiene }\end{array}$ & $\begin{array}{l}\text { Antiphl. Thera- } \\
\text { pie Sattelge- } \\
\text { lenks-Schiene }\end{array}$ & $\begin{array}{l}\text { Antiphl. Thera- } \\
\text { pie Sattelge- } \\
\text { lenks-Schiene }\end{array}$ & $\begin{array}{l}\text { Mittelwert } \\
\text { und Standard- } \\
\text { abweichung }\end{array}$ & $\begin{array}{l}\text { Signifikanz } \\
\text { beim Vergleich } \\
\text { zu präoperativ }\end{array}$ \\
\hline \multicolumn{8}{|l|}{ Schmerz VAS } \\
\hline preoperativ & 6 & 7 & 7 & 9 & 8 & $7,4 \pm 1,1$ & \\
\hline post 1 Monat & 3 & 2 & 0 & 3 & 3 & $2,2 \pm 1,3$ & $p=0,042$ \\
\hline post 3 Monate & 0 & 7 & 2 & 3 & 0 & $2,4 \pm 2,9$ & nicht signifikant \\
\hline \multicolumn{8}{|c|}{ Schmerz VAS Ruhe } \\
\hline preoperativ & 1 & 6 & 7 & 5 & 0 & $3,8 \pm 3,1$ & \\
\hline post 1 Monat & 0 & 0 & 0 & 0 & 0 & $0,0 \pm 0,0$ & nicht signifikant \\
\hline post 3 Monate & 0 & 4 & 0 & 0 & 0 & $0,8 \pm 1,8$ & nicht signifikant \\
\hline \multicolumn{8}{|l|}{ Grobkraft Ball 5} \\
\hline preoperativ & 0,3 & 0,4 & 0,45 & 0,2 & 0,4 & $0,35 \pm 0,10$ & \\
\hline post 1 Monat & 0,3 & 0,5 & 0,5 & 0,3 & 0,4 & $0,40 \pm 0,10$ & nicht signifikant \\
\hline post 3 Monate & 0,4 & 0,5 & 0,5 & 0,3 & 0,4 & $0,42 \pm 0,08$ & nicht signifikant \\
\hline \multicolumn{8}{|c|}{ Pinch Griff Ball 2} \\
\hline preoperativ & 0,2 & 0,25 & 0,25 & 0,3 & 0,3 & $0,26 \pm 0,04$ & \\
\hline post 1 Monat & 0,2 & 0,4 & 0,3 & 0,25 & 0,25 & $0,28 \pm 0,08$ & nicht signifikant \\
\hline post 3 Monate & 0,35 & 0,25 & 0,45 & 0,25 & 0,25 & $0,31 \pm 0,09$ & nicht signifikant \\
\hline \multicolumn{8}{|l|}{ DASH } \\
\hline preoperativ & 46 & 79 & 61 & 62 & 44 & $58 \pm 14$ & \\
\hline post 1 Monat & 23 & 54 & 39 & 39 & 26 & $36 \pm 12$ & $p=0,042$ \\
\hline post 3 Monate & 23 & 72 & 39 & 29 & 1 & $33 \pm 26$ & $p=0,043$ \\
\hline
\end{tabular}


sche Operationstechniken trotz im Allgemeinen hoher Erfolgsrate zu einer irreversiblen Veränderung der Handwurzelanatomie führen.

Wenngleich wir bisher nur vorläufige Ergebnisse von lediglich 5 Patienten präsentieren, kann hier doch bereits ein positiver Trend bezüglich der Schmerzreduktion und Kraftentwicklung abgelesen werden. Hier könnte der sofort vorhandene Gleiteffekt des Fettgewebes im arthrotischen Gelenk zu einer Minderung der Beschwerden zu führen. Ob der darüber hinaus erhoffte regenerative Effekt der co-transplantierten Stammzellen hierbei eine Rolle spielt, ist noch nicht abzusehen. Hypothetisch erscheint unter den Bedingungen, welche in einem degenerativ veränderten Gelenk herrschen (Knorpelapoptose) die Aktivierung einer chondrogenen Differenzierung denkbar. Diese Vermutung wird durch aktuelle Arbeiten über Knorpelregeneration durch adipose derived stem cells unterstützt $[9,10]$.

Modifizierte Fettgewebstransplantate oder etwa Stammzellsuspensionen, welche aus Fettgewebe hergestellt wurden, zu verwenden, gestaltet sich in Deutschland jedoch schwierig. Die Herstellung und Anwendung zelltherapeutischer Präparate wird in Deutschland mit dem Arzneimittelgesetz geregelt. Karagianni et al. haben 2013 eine umfassende Übersichtsarbeit publiziert, welche den aktuellen gesetzlichen Rahmen abbildet [11]. Fettgewebsstammzellen werden als „Arzneimittel für neuartige Therapien“ eingestuft und hier ist eine Herstellungserlaubnis nach §13 Arzneimittelgesetz notwendig. Konventionelle Lipoaspirate enthalten Fettstammzellen [12]. Werden diese Lipoaspirate aber ohne industrielles Herstellungsverfahren, unter Einhaltung etablierter Herstellungsschritte und ohne substanzielle Verarbeitung als Transplantate angewendet, so gelten sie als klassische Gewebezubereitung und ihr Einsatz bedarf keiner Genehmigung [11]. Hier liegt der entscheidende Vorteil klassischer autologer Fetttransplantate. Die Coleman Technik ist ein, wenn nicht das Standardverfahren der autologen Fetttransplantation [13], die Ausbeute vitaler Fettzellen ist gegenüber Filtertechniken aufgrund der zentrifugationsbedingten Kondensation des Lipoaspirates überlegen [14]. Es konnte gezeigt werden, dass verschiedenste Absaug-, und Aufbereitungstechniken zu stammzellreichen Fettgewebstransplantaten führen [15]. Bei der Untersuchung von zentrifugationsbedingt kondensierten Lipoaspiraten konnte der höchste Anteil von Vorläuferzellen in der Phase der höchsten Dichte nachgewiesen werden [16]. Eine Verwendung von prozessiertem Fettgewebe oder isolierte Stammzellfraktionen ist aus unserer Sicht nicht zwingend notwendig, da bereits klassische Lipoaspirate eine ausreichende StammzellStromazellfraktion enthalten. Da klassische Lipoaspirate anolog zu Hyaluronsäure viskosupplementäre Eigenschaften aufweisen, erscheint ihre Verwendung gegenüber Fettstammzellen nicht nur unter den herrschenden gesetzlichen Rahmenbedingung schlüssiger, sondern auch weil sie eine Gleitfunktion im verschlissenen Gelenk ausüben können.

Ein Gleiteffekt intraartikulär injizierter Hyaluronsäure konnte nach Handgelenksarthroskopien eine signifikant bessere Schmerzreduktion als eine alleinige Handgelenksarthroskopie erzielen [17]. Hyaluronsäure schützt und bedeckt Gelenkoberflächen [18]. In einer prospektiven randomisiert doppelblinden Studie konnte ein im Vergleich zum Zeitpunkt vor Injektion signifikant verringerter Schmerz 4 Wochen nach Injektion von Hyaluron in das Daumensattelgelenk nachgewiesen werden [19]. Dieser Effekt trat allerdings auch in der Plazebogruppe (Kochsalzlösung) auf. Mit Kortison zeigte sich der Effekt nur 2 Wochen post injectionem. Nach 12 Wochen war nur noch Hyaluronsäure in der Lage die Schmerzen im Vergleich zum Zeitpunkt vor der Injektion zu mildern, nicht jedoch Kochsalzlösung oder Kortison. Leider sind die Ergebnisse dieser Studie nicht direkt mit unseren Ergebnissen zu vergleichen, da der Ausgangs DASH mit $33 \pm 5$ in der Kontrollgruppe, mit $41 \pm 4$ in der Kortisongruppe und mit $37 \pm 4$ Hyaluronsäuregruppe deutlich niedriger als bei den von uns mit autologer Fetttransplantation ( $58 \pm 14$ ) behandelten Patienten lag. Auch lagen die Schmerzen vor den jeweiligen Injektionen mit VAS $5 \pm 1$ auf einem deutlich geringeren $\mathrm{Ni}$ veau (vor autologer Fetttransplantation 7,4 $\pm 1,1$ ). Für den Verlauf werden in dieser Studie von Heywoths et al. weiterhin nur Flussdiagramme und keine exakten Werte angegeben. Letztendlich werden die Stadien der Rhizarthrose ebenfalls nicht genannt.

Durchschnittlich 27 Monate nach operativer Versorgung mittels Trapezektomie und Kapselraffung konnten Schibli-Beer et al. einen durchschnittlichen DASH Wert von $29 \pm 22$ und eine Schmerzintensität von 1,8 $\pm 2,0$ nach VAS ermitteln [20]. Die frühen Ergebnisse nach autologer Fetttransplantation sind hier mit einem durchschnittlichen DASH Wert von $32 \pm 26$ und Schmerzen nach VAS von $2,4 \pm 2,9$ bei Belastung und $0,8 \pm 1,8$ in Ruhe vergleichbar, wenn auch berücksichtigt werden muss, dass es sich hierbei um frühe postoperative Ergebnisse handelt.

Etwas bessere Werte präsentierten Wittemann et al. durchschnittlich 34 Monate nach Epping Plastik mit einem DASH Wert von 26 [21]. Rab et al. verglichen die Verwendung verschiedener Sehnen zur Suspensionsarthroplastik. Bei Verwendung der FCR Sehne zeigten sich nach durchschnittlich 31 Monaten ein DASH Wert von $29 \pm 16$ und eine Schmerzintensität von $0,9 \pm 1,5$ und bei Verwendung der APL Sehne zeigten sich nach durchschnittlich 23 Monaten ein DASH Wert von $23 \pm 12$ und eine Schmerzintensität von 1,1 $\pm 1,7$ [22].

Anzumerken ist, dass Patientin 2 unserer Studie eine Mittelfußfraktur 6 Wochen nach autologer Fettinjektion in das Sattelgelenk erlitt. Sie musste fortan an Unterarmgehstützen laufen, also bis zum Untersuchungszeitpunkt 3 Monate postoperativ für 6 Wochen. Dieses stellt eine hohe Belastung für ein arthrotisches Sattelgelenk dar. Ihr DASH Score 3 Monate postoperativ war daher mit 72 vergleichsweise hoch, ebenso hatte sie 3 Monate postoperativ Schmerzen nach VAS von 7 bei Belastung und 4 in Ruhe. Einen Monat postoperativ hatten die Werte hier noch 2 bei Belastung und 0 in Ruhe betragen. Hier zeigen sich Grenzen der Technik. Eine zu große Belastung gefährdet nach Eigenfettinjektion das Behandlungsergebnis. Es ist zu diskutieren in wie fern ein Patient nach Trapezektomie zu diesem Zeitpunkt überhaupt an Unterarmgehstützen hätte gehen können. Unter Ausschluss dieser Patientin beträgt der durchschnittliche Schmerz der übrigen Patienten 3 Monate postoperativ bei Belastung 1,3 und in Ruhe 0, der DASH Score nur 23.

Eine Schwäche der Arbeit ist sicherlich, dass es sich hierbei um eine Pilotstudie ohne Randomisierung und Kontrollgruppe handelt. Daher können aus den ersten Ergebnissen allenfalls erste Trends abgelesen werden. Auch wenn die frühen Ergebnisse nach autologer Fetttransplantation in das Daumensattelgelenk durchaus mit etablierten Techniken vergleichbar sind, müssen sie natürlich unter diesem Hintergrund betrachtet werden. Darüber hinaus kann zum aktuellen Zeitpunkt auch ein Plazeboeffekt nicht sicher ausgeschlossen werden.

In Anbetracht der geringen Invasivität und der deutlichen Beschwerdebesserung,stelltdieautologeFettgewebstransplantation in das Daumensattelgelenk eine interessante Alternative dar. Ein besonderer Vorteil besteht in der Tatsache, dass bei einem 
Therapieversagen die klassischen handchirurgischen Techniken unverändert zur Verfügung stehen. Dieses ist im Falle einer implantierten Prothese oder einer bereits erfolgten Trapezektomie nicht sicher gegeben.

Es bleibt abzuwarten, ob nach Untersuchung eines größeren Patientengutes über einen längeren Zeitraum die hier vorgestellte Technik ihren Platz unter den Standardeingriffen finden wird.

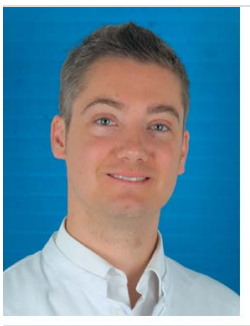

\section{Christian Herold}

Von 1998 bis 2004 Studium der Humanmedizin in Hannover mit Auslandsaufenthalten in Charlottesville/USA und Hiroshima/Japan. Von 2002 bis 2004 Studium der Zahnmedizin in Hannover. Promotion: Chordome und Chondrosarkome der Schädelbasis - retrospektive Analyse einer operativen Serie. Von 2005 bis 2006 Assistenzarzt in der Neurochirurgie, bei Prof. Dr. Samii am INI in Hannover, ab Sommer 2006 Assistenzarzt in der Abteilung für Plastische-, Hand und Wiederherstellungschirurgie, bei Prof. Dr. P. M. Vogt. 2011 Facharzt für Plastische und Ästhetische Chirurgie. 2012 Habilitation unter Prof. Dr. P. M. Vogt an der MHH in Hannover mit dem Thema: Vitalitätsuntersuchungen und Volumenerhalt bei der autologen Fetttransplantation. Seit 2012 Leitender Oberarzt bei Dr. S. Allert im Sana Klinikum Hameln Pyrmont.

Interessenkonflikt: Nein

\section{Literatur}

1 Armstrong AL, Hunter JB, Davis TR. The prevalence of degenerative arthritis of the base of the thumb in post-menopausal women. J Hand Surg Br 1994; 19: 340-341

2 Cook GS, Lalonde DH. MOC-PSSM CME article: Management of thumb carpometacarpal joint arthritis. Plast Reconstr Surg 2008; 121: 1-9

3 Ellis CV, Kulber DA. Acellular dermal matrices in hand reconstruction. Plast Reconstr Surg 2012; 130: 256S-269S

4 Hohendorff B, Staub L, Kaiser T et al. Working ability after tendon interposition arthroplasty for degenerative arthritis of the thumb trapeziometacarpal joint. Handchir Mikrochir Plast Chir 2008; 40: 175-181

5 Ingegnoli F, Soldi A, Meroni PL. Power Doppler sonography and clinical monitoring for hyaluronic Acid treatment of rhizarthrosis: a pilot study. J Hand Microsurg 2011; 3: 51-54

6 Huang JI, Zuk PA, Jones NF et al. Chondrogenic potential of multipotential cells from human adipose tissue. Plast Reconstr Surg 2004; 113: 585-594
7 Zuk PA, Zhu M, Ashjian P et al. Human adipose tissue is a source of multipotent stem cells. Mol Biol Cell 2002; 13: 4279-4295

8 ter Huurne $M$, Schelbergen $R$, Blattes $R$ et al. Antiinflammatory and chondroprotective effects of intraarticular injection of adipose-derived stem cells in experimental osteoarthritis. Arthritis Rheum 2012; 64: 3604-3613

9 Wu L, Cai X, Zhang S et al. Regeneration of articular cartilage by adipose tissue derived mesenchymal stem cells: perspectives from stem cell biology and molecular medicine. J Cell Physiol 2013; 228: 938-944

10 Toupet K, Maumus M, Peyrafitte JA et al. Long-term detection of human adipose-derived mesenchymal stem cells after intraarticular injection in SCID mice. Arthritis Rheum 2013; 65: 1786-1794

11 Karagianni M, Kraneburg $U$, Kluter $\mathrm{H}$ et al. Autologous fat grafts and supportive enrichment with adipose tissue stromal cells. Handchir Mikrochir Plast Chir 2013; 45: 93-98

12 Brayfield C, Marra K, Rubin JP. Adipose stem cells for soft tissue regeneration. Handchir Mikrochir Plast Chir 2010; 42: 124-128

13 Rennekampff HO, Reimers K, Gabka CJ et al. Current perspective and limitations of autologous fat transplantation-"consensus meeting" of the German Society of Plastic, Reconstructive and Aesthetic Surgeons at Hannover; September 2009. Handchir Mikrochir Plast Chir 2010; 42: $137-142$

14 Herold C, Pflaum M, Utz P et al. Viability of autologous fat grafts harvested with the Coleman technique and the tissu trans system (shippert method): a comparative study. Handchir Mikrochir Plast Chir 2011; 43: 361-367

15 Fisher C, Grahovac TL, Schafer ME et al. Comparison of harvest and processing techniques for fat grafting and adipose stem cell isolation. Plast Reconstr Surg 2013; 132: 351-361

16 Allen RJJr, Canizares O Jr, Scharf C et al. Grading lipoaspirate: is there an optimal density for fat grafting? Plast Reconstr Surg 2013; 131: 38-45

17 Schutz A, Dobner P. Effect of wrist arthroscopy with intraarticular hyaluronan substitution therapy: a randomised, controlled, prospective, non-blinded, single-centre, comparative trial. Handchir Mikrochir Plast Chir 2013; 45: 277-284

18 Hempfling $H$. Intra-articular hyaluronic acid after knee arthroscopy: a two-year study. Knee Surg Sports Traumatol Arthrosc 2007; 15: 537-546

19 Heyworth BE, Lee JH, Kim PD et al. Hylan versus corticosteroid versus placebo for treatment of basal joint arthritis: a prospective, randomized, double-blinded clinical trial. J Hand Surg Am 2008; 33: 40-48

20 Schibli-Beer S, Mark G, Canova M. Results after simple trapeziectomy and capsular fixation for osteoarthritis of the trapeziometacarpal joint. Handchir Mikrochir Plast Chir 2008; 40: 169-174

21 Wittemann M, Demir E, Sauerbier $M$ et al. The Epping resection-suspension arthroplasty procedure. A standard procedure in the operative treatment of trapeziometacarpal osteoarthrosis? Handchir Mikrochir Plast Chir 2002; 34: 49-58

22 Rab M, Gohritz A, Gohla T et al. Long-term results after resection arthroplasty in patients with arthrosis of the thumb carpometacarpal joint: comparison of abductor pollicis longus and flexor carpi radialis tendon suspension. Handchir Mikrochir Plast Chir 2006; 38: 98-103 